\title{
Antibody responses to endemic coronaviruses modulate COVID-19 convalescent plasma functionality
}

\author{
William R. Morgenlander, ${ }^{1}$ Stephanie N. Henson, ${ }^{1}$ Daniel R. Monaco, ${ }^{1}$ Athena Chen, ${ }^{2}$ Kirsten Littlefield, ${ }^{3}$ Evan M. Bloch, ${ }^{4}$ \\ Eric Fujimura, ${ }^{5}$ Ingo Ruczinski, ${ }^{2}$ Andrew R. Crowley, ${ }^{6}$ Harini Natarajan, ${ }^{6}$ Savannah E. Butler, ${ }^{6}$ Joshua A. Weiner, ${ }^{7}$ Mamie Z. Li, ${ }^{5}$ \\ Tania S. Bonny, ${ }^{4}$ Sarah E. Benner, ${ }^{4}$ Ashwin Balagopal, ${ }^{8}$ David Sullivan, ${ }^{3,8}$ Shmuel Shoham, ${ }^{8}$ Thomas C. Quinn, ${ }^{8,9}$ \\ Susan H. Eshleman, ${ }^{4}$ Arturo Casadevall, ${ }^{3}$ Andrew D. Redd, ${ }^{8,9}$ Oliver Laeyendecker, ${ }^{8,9}$ Margaret E. Ackerman, ${ }^{7}$ Andrew Pekosz, ${ }^{3}$ \\ Stephen J. Elledge, ${ }^{5}$ Matthew Robinson, ${ }^{8}$ Aaron A.R. Tobian, ${ }^{4}$ and H. Benjamin Larman ${ }^{1}$
}

Institute for Cell Engineering, Division of Immunology, Department of Pathology, Johns Hopkins University School of Medicine, Baltimore, Maryland, USA. ²Department of Biostatistics, and ${ }^{3} \mathrm{~W}$. Harry Feinstone Department of Molecular Microbiology and Immunology, Johns Hopkins University Bloomberg School of Public Health, Baltimore, Maryland, USA. ${ }^{4}$ Division of Transfusion Medicine, Department of Pathology, Johns Hopkins University School of Medicine, Baltimore, Maryland, USA. 'Division of Genetics, Department of Medicine, Howard Hughes Medical Institute, Brigham and Women's Hospital, and Department of Genetics, Program in Virology, Harvard Medical School, Boston, Massachusetts, USA. ${ }^{6}$ Department of Microbiology and Immunology, Geisel School of Medicine, Dartmouth College, Hanover, New Hampshire, USA. TThayer School of Engineering, Dartmouth College, Hanover, New Hampshire, USA. ${ }^{8}$ Division of Infectious Diseases, Department of Medicine, Johns Hopkins University School of Medicine, Baltimore, Maryland, USA. ${ }^{9}$ Division of Intramural Research, National Institute of Allergy and Infectious Diseases (NIAID), NIH, Bethesda, Maryland, USA.

SARS-CoV-2 (CoV2) antibody therapies, including COVID-19 convalescent plasma (CCP), monoclonal antibodies, and hyperimmune globulin, are among the leading treatments for individuals with early COVID-19 infection. The functionality of convalescent plasma varies greatly, but the association of antibody epitope specificities with plasma functionality remains uncharacterized. We assessed antibody functionality and reactivities to peptides across the CoV2 and the 4 endemic human coronavirus (HCoV) genomes in 126 CCP donations. We found strong correlation between plasma functionality and polyclonal antibody targeting of CoV2 spike protein peptides. Antibody reactivity to many HCoV spike peptides also displayed strong correlation with plasma functionality, including pan-coronavirus cross-reactive epitopes located in a conserved region of the fusion peptide. After accounting for antibody crossreactivity, we identified an association between greater alphacoronavirus NL63 antibody responses and development of highly neutralizing antibodies against CoV2. We also found that plasma preferentially reactive to the CoV2 spike receptor binding domain (RBD), versus the betacoronavirus HKU1 RBD, had higher neutralizing titer. Finally, we developed a 2-peptide serosignature that identifies plasma donations with high anti-spike titer, but that suffer from low neutralizing activity. These results suggest that analysis of coronavirus antibody fine specificities may be useful for selecting desired therapeutics and understanding the complex immune responses elicited by CoV2 infection.

\section{Introduction}

Coronaviruses cause human respiratory diseases that range from asymptomatic to fatal. Endemic human coronaviruses (HCoVs)

Authorship note: WM and SH contributed equally to this work. MR, AART, and BL are co-senior authors.

Conflict of interest: HBL and SJE are inventors on a patent application (US20160320406A, "Detection of an antibody against a pathogen") filed by Brigham and Women's Hospital that covers the use of the VirScan technology to identify pathogen antibodies and are founders of ImmuneID. HBL is a founder of Alchemab and Portal Bioscience, and is an advisor to CDI Laboratories and TSCAN Therapeutics. SJE is a founder of TSCAN Therapeutics, MAZE Therapeutics, and Mirimus. SJE serves on the scientific advisory board of Homology Medicines, TSCAN Therapeutics, MAZE, XChem, and is an advisor for MPM. SS has received grants from Ansun, Astrellas, Cidara, F2G, Merck, T2, Reviral, Shire, Shionogi, and Scynexis. SS has received personal fees from Acidophil, Amplyx, Janssen, Merck, Reviral, Karyopharm, Intermountain Health, and Immunome.

Copyright: () 2021, American Society for Clinical Investigation

Submitted: December 17, 2020; Accepted: February 3, 2021; Published: April 1, 2021

Reference information: J Clin Invest. 2021;131(7):e146927.

https://doi.org/10.1172/JCl146927. that cause the common cold include 2 alphacoronaviruses (229E and NL63) and 2 betacoronaviruses (OC43 and HKU1). Middle Eastern respiratory syndrome (MERS) coronavirus and the severe acute respiratory syndrome coronavirus (SARS-CoV-1) are betacoronaviruses that can cause severe pneumonia. In late 2019, a novel severe pneumonia-causing betacoronavirus, SARS-CoV-2 (CoV2), was described in Wuhan, China. As of March 2021, the COVID-19 pandemic, caused by the spread of CoV2, had infected over 120 million individuals and resulted in over 2.6 million deaths worldwide (1).

There is growing evidence for the potential of anti-CoV2 antibodies to treat COVID-19. CoV2 antibodies have been administered in the form of monoclonal antibodies directed at specific CoV2 epitopes and hyperimmune globulin or COVID-19 convalescent plasma (CCP) obtained from individuals who recovered from COVID-19 (2-5). CCP has received Emergency Use Authorization from the United States Food and Drug Administration (FDA) for treatment of COVID-19. Efficacy data for CCP are mixed, but recent publications suggest CCP is most 
A

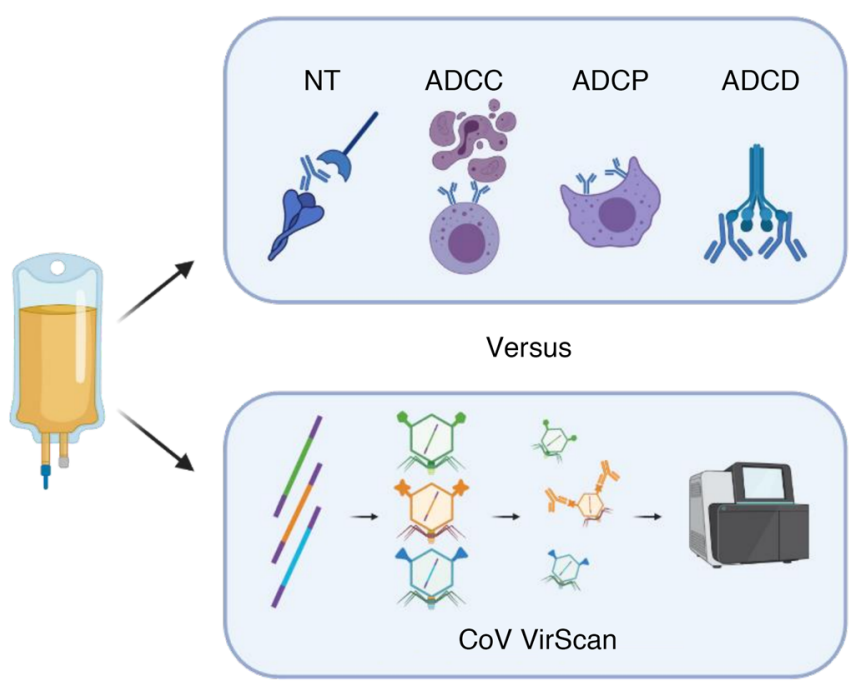

B
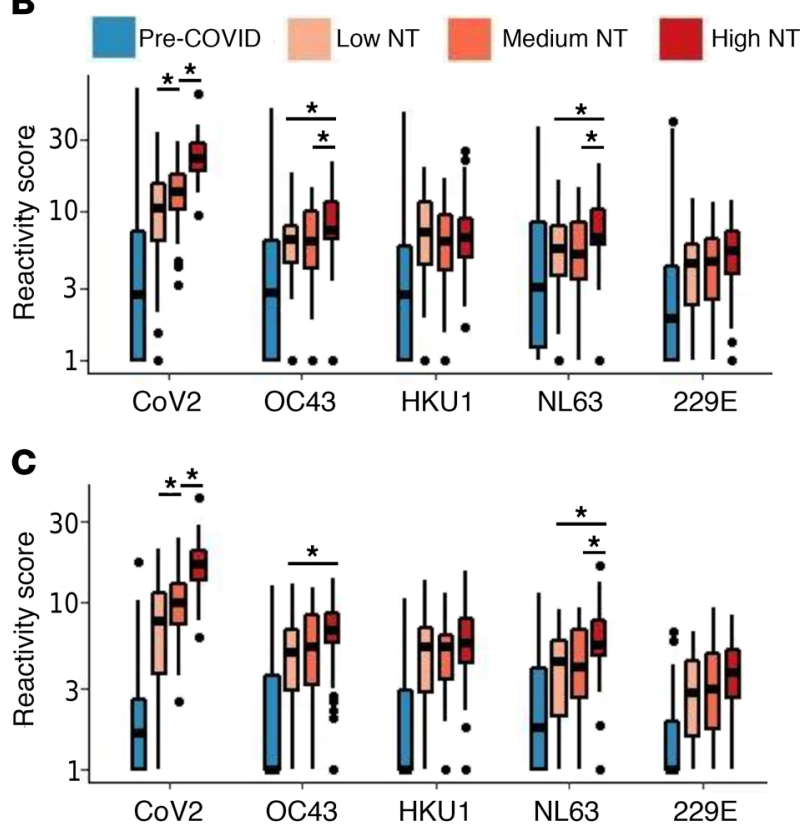

Figure 1. Correlating coronavirus peptide reactivity and functionality of COVID-19 convalescent plasma. (A) One hundred twenty-six eligible COVID-19 convalescent plasma donors underwent functional analysis and antibody profiling via VirScan with a comprehensive coronavirus (CoV) peptide library. Functionalities included neutralizing titer (NT), antibody-dependent cellular cytotoxicity (ADCC), antibody-dependent cellular phagocytosis (ADCP), and antibody-dependent complement deposition (ADCD). Plasma from 87 prepandemic controls were additionally analyzed via VirScan. (B) COVID-19 convalescent plasma was divided into groups based on neutralizing titer area under the curve (NT AUC: Low NT, $<40$ [ $n=55$ ]; Medium NT, 40 to 160 [ $n=39$ ]; and High NT, $\geq 160$ [ $n=32]$ ). Aggregate virus score was calculated as the sum of all log-transformed fold changes of peptides designed for a given virus. Bars with an asterisk indicate convalescent plasma groups that had significantly different scores. (C) Aggregate virus scores from peptides defining dominant regions. ${ }^{*} P<0.05$ by 2 -sided Wilcoxon's test.

effective when provided early in disease course and specifically when units contain high titers of CoV2 antibodies $(6,7)$.

The antibody response to CoV2 is highly variable in terms of titer $(8,9)$, avidity $(10)$, antigenic preference $(11,12)$, kinetics of induction (9), isotype usage (13), and functionally protective capacity (13). Differential preexisting immune responses to endemic HCoVs may contribute to the large variation in CoV2 antibody response. Recent studies of prepandemic plasma identified a low prevalence of preexisting reactivity against the $\mathrm{S} 2$ subunit of the CoV2 spike (S) protein $(13,14)$. S2 contains structures that are critical for virus entry into cells, such as the fusion peptide (FP), which is conserved across coronaviruses; sequence conservation in this region may explain the presence of these CoV2-reactive antibodies prior to the COVID-19 pandemic (12). Boosting of preexisting $\mathrm{HCoV}$ antibodies in response to infection with CoV2 may occur in the absence of antibody functionality, a phenomenon referred to as "original antigenic sin" (15). Alternatively, $\mathrm{HCoV}$ antibody responses may prove beneficial during $\mathrm{CoV} 2$ infection, as $\mathrm{HCoV}$ neutralization activity has been correlated with decreased disease severity (16), and anti-CoV2 activity of preexisting $\mathrm{HCoV}$ antibodies has been suggested (14). There remains an important gap in understanding the relationships between cross-reactive $\mathrm{HCoV}$ antibodies, CoV2 antibody binding specificities, and the functional activities of CoV2 antibodies.

In this study, we used systems serology and massively multiplexed epitope profiling to characterize the functionality and fine specificities of coronavirus antibodies in a cohort of eligible CCP donors (Figure 1A). We correlated dominant CoV2 and $\mathrm{HCoV}$ peptide reactivities with viral neutralization, antibody-dependent cellular phagocytosis (ADCP), antibody-dependent cellular cytotoxicity (ADCC), and antibody-dependent complement deposition (ADCD). We developed an algorithm to deconvolute crossreactivity among homologous peptides, which helped explain how disparate $\mathrm{HCoV}$ antibody responses may modulate functional characteristics of $\mathrm{CoV} 2$ antibodies.

\section{Results}

Systems serology and VirScan analyses of convalescent plasma donations. CCP for this study were provided by 126 eligible convalescent donors for therapeutic use 13-67 days after PCRconfirmed CoV2 infection $(8,10)$. As part of a CCP treatment trial, CoV2 neutralizing titer (NT) was measured for each sample and reported as an area under the curve (NT AUC). We divided CCP into groups according to their neutralizing activity: High NT (NT AUC $\geq 160, n=32$ ), Medium NT (NT AUC $\geq 40$ and $<160, n$ $=39$ ), and Low NT (NT AUC $<40, n=55)(8)$. We also measured ADCP, ADCC, and ADCD for each plasma donation (13). We recently used a massively multiplexed antibody profiling system (VirScan) to analyze peptide epitopes across over 230 COVID-19 and 190 prepandemic samples (12). VirScan is a programmable phage display technology that can be used to evaluate the binding specificity of plasma antibodies. The coronavirus VirScan library contains 3,466 peptides, each 56 amino acids in length, that tile across the CoV2 and all 4 endemic HCoV genomes with 28- 
A
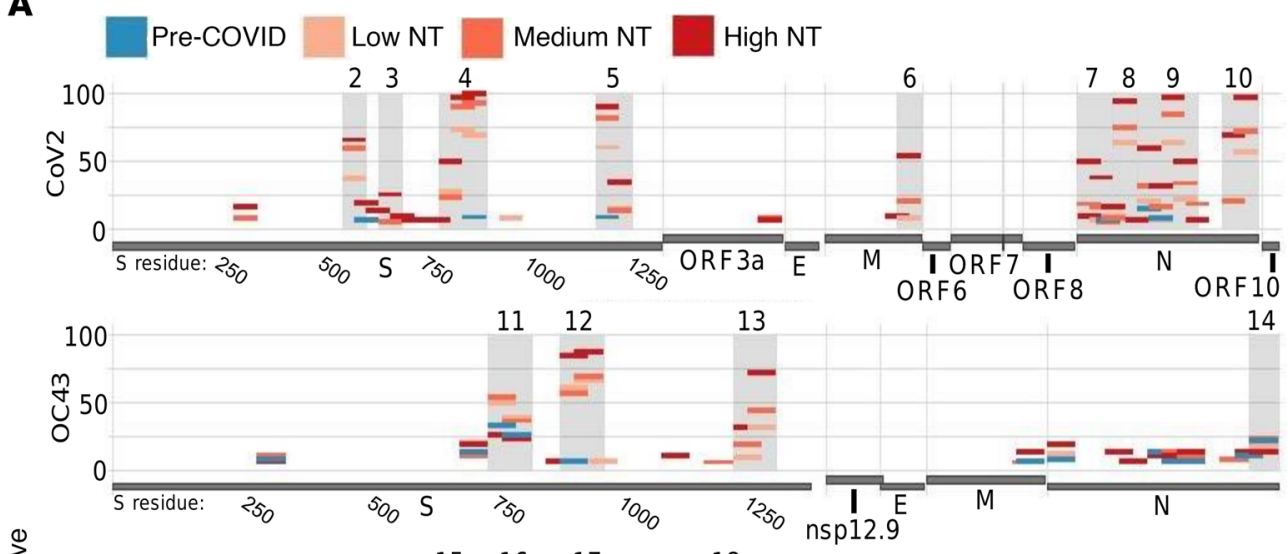

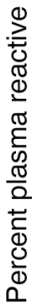
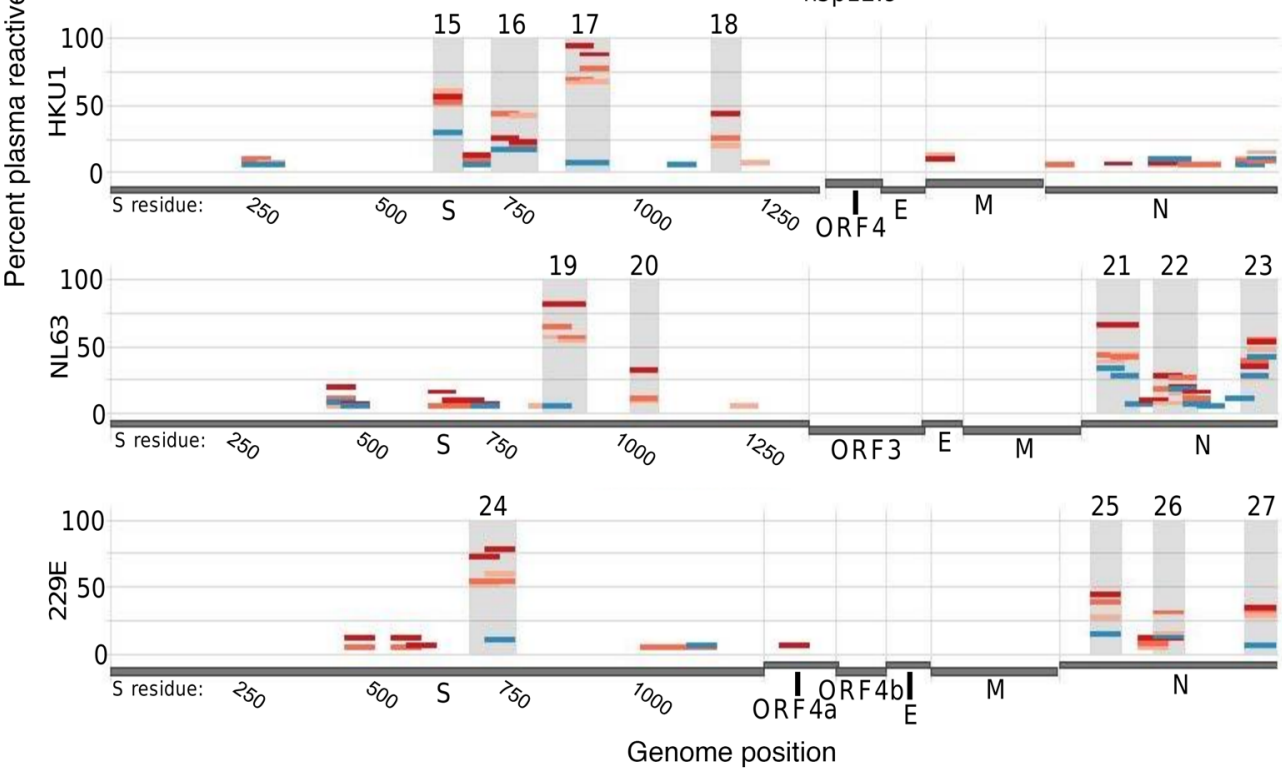

B
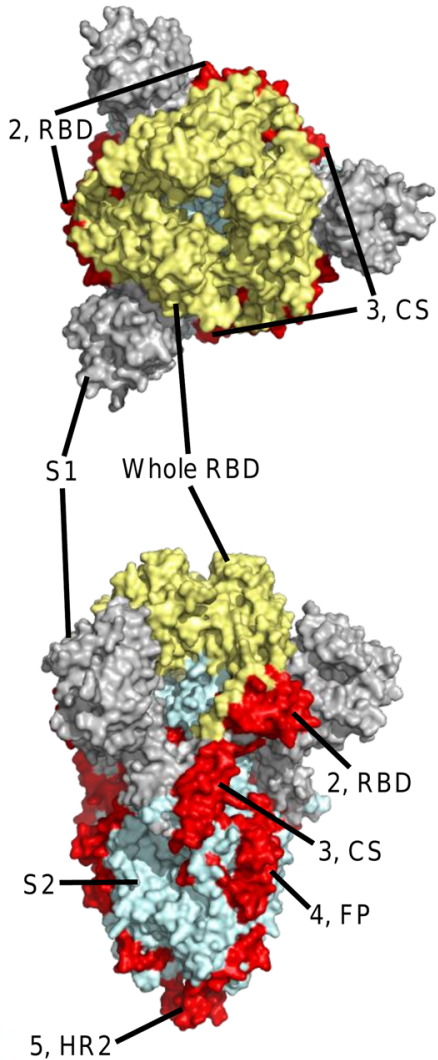

Figure 2. Defining coronavirus peptide epitopes targeted by COVID-19 convalescent plasma. (A) The percentages of samples in each group (Pre-COVID, $n=$ 87; Low NT, $n=55$; Medium NT, $n=39$; and High NT, $n=32$ ) with reactivity to a particular peptide were plotted according to the peptide's position along each viral genome. Dominant regions are shaded. Amino acid residue number is included for the spike protein (S). Full genome plots are provided in Supplemental Figure 1. Gene products: spike (S), envelope (E), membrane (M), and nucleocapsid (N). (B) Immunodominant regions of CoV2 S are mapped onto the CoV2 $\mathrm{S}$ structure (19). Trimeric spike is shown with the whole receptor binding domain (RBD) in yellow, the rest of S1 in gray, and S2 in blue. Immunodominant regions: 2 or RBD, 3 or S1/S2 cleavage site (CS), 4 or fusion peptide (FP), and 5 or heptad repeat 2 (HR2).

amino acid overlaps; the library also covers SARS-CoV-1, MERS, and 3 related bat coronaviruses: BatCoV-Rp3, BatCoV-HKU3, and BatCoV-279 (12). At several genomic locations, single amino acid variants of CoV2 are also represented in the library. We used this coronavirus VirScan library to identify the epitopes targeted in each of the 126 CCP samples. For comparison, we also performed coronavirus VirScan on 87 pre-COVID-19 pandemic (Pre-COVID) plasma samples (17).

For all plasma samples, we measured antibody binding to each 56-amino acid peptide in the library. In each group of samples, we examined 2 virus-level reactivity scores: (a) aggregate reactivity across all peptides from each virus (Figure 1B), and (b) aggregate reactivity across the frequently reactive (dominant) peptides from each virus (Figure 1C). Dominant peptides were defined using a threshold of $20 \%$ reactivity in any sample group. The aggregate CoV2 scores were highest in High NT CCP, as expected. High NT CCP also exhibited significantly greater aggregate NL63 and OC43 reactivity compared with Low NT CCP, potentially reflecting some degree of crossreactivity with CoV2. In addition, a higher number of total reactivities (polyclonality) in the dominant regions of $\mathrm{CoV} 2$ and NL63 correlated with higher NT AUC (Supplemental Figure 1; Pearson's correlations: CoV2 $P<1 \times 10^{-8}$, NL63 P = 0.02; supplemental material available online with this article; https:// doi.org/10.1172/JCI146927DS1).

Next, we examined the specific epitopes targeted by antibodies in CCP (Figure 2A and Supplemental Figure 2). Using the $20 \%$ reactivity threshold, we identified 27 regions of dominant reactivity that largely corresponded to regions we had previously established (12). Among the samples that harbored dominant reactivities, the magnitude of reactivity to specific peptides was not associated with NT AUC, with one exception: reactivity to an immunodominant region that overlaps the CoV2 spike (S) receptor binding domain (RBD) (residues 533-588) was greater in High NT CCP (Supplemental Figure 3; Wilcoxon's test: $P=0.014$ vs. Medium NT, $P<0.001$ vs. Low NT). At the peptide level, it there- 
fore appears that the response clonality, defined by the number of reactive epitopes, rather than the magnitude of reactivity to individual peptides, distinguishes Low NT from High NT CCP.

Table 1 provides the genomic location and reactivity profiles for each immunodominant peptide (amino acid sequences in Supplemental Table 1). Peptides with identical start and stop locations represent genomic variants. Three regions of CoV2 S, 1 region of the CoV2 membrane protein (M), and 4 regions of the CoV2 nucleocapsid (N) protein were recognized by $50 \%-100 \%$ of High NT CCP. Antibodies in Medium NT and Low NT CCP recognized many of the same regions, but at a lower frequency. Exceptions to this pattern were that Low NT CCP displayed the most frequent reactivity to peptides around the S1/S2 cleavage site (CS) from the betacoronaviruses HKU1 and OC43. Many HCoV dominant regions showed increasing frequency of reactivity with increasing NT AUC, including the highly conserved S FP, which is required for entry of coronaviruses into host cells. We previously identified the CoV2 FP as one of the most reactive public CoV2 epitopes; the corresponding peptide from $\mathrm{HCoV}$ viruses show high conservation and boosting in CoV2 patients (12). Peptide reactivities associated with NT AUC frequently correlated with other plasma functionalities (Table 1); this was not surprising, since these functionalities were all independently correlated with NT AUC (13). Interestingly, the peptide reactivity that had the strongest correlation with ADCC derives from an intravirion portion of CoV2 M (residues 169-224), which is a known T cell epitope (18). For structural context, we mapped the dominant $\mathrm{CoV} 2 \mathrm{~S}$ regions onto the mature folded S protein structure (Figure 2B) (19).

CoV2 and endemic coronavirus cross-reactivity. Many immunodominant $\mathrm{CoV} 2$ peptides are homologous to $\mathrm{HCoV}$ peptides, and $\mathrm{HCoV}$ peptides were more frequently recognized by the CCP compared with Pre-COVID plasma. We therefore evaluated potential cross-reactivity among dominant epitopes. We identified 2 independent clusters of peptides from $\mathrm{CoV} 2$ and the $\mathrm{HCoVs}$ that exhibited significantly correlated reactivities (Figure 3A). The largest cluster was composed of highly correlated reactivities to FP from the S2 subunit of each HCoV S; all of these peptides showed sequence homology to the immunodominant CoV2 FP peptides (Figure 3B). The immunodominant FP peptides were the most frequently recognized peptides from each coronavirus among all CCP NT groups (Table 1). Plasma with antibodies against CoV2 FP most often also reacted with all other coronavirus FPs, including the zoonotic bat coronaviruses represented in our library (Supplemental Figure 4). The smaller cluster of correlated reactivity in Figure 3A contained a CoV2 S HR2 peptide and the homologous OC43 S HR2 peptides. Of note, CoV2 FP and HR2 peptides were targeted by antibodies in up to $8 \%$ of Pre-COVID plasma. This suggests that the immunodominant $\mathrm{CoV} 2 \mathrm{FP}$ and HR2 regions may be key targets of preexisting $\mathrm{HCoV}$ antibodies that may provide heterologous protection from $\mathrm{CoV} 2$ infection and/or are boosted during CoV2 antibody responses.

We next evaluated the relationships between CoV2 peptides and homologous $\mathrm{HCoV}$ peptides by comparing the magnitudes of the reactivities towards the dominant CS, RBD, and FP peptides from CoV2 and HKU1 (Figure 3, C-E). The 2 FP peptides (CoV2 residues 813-868, HKU1 residues 869-924) had significant amino acid-level homology and a strong positive correlation in antibody reactivity regardless of sample group (including PreCOVID). Meanwhile, reactivity to the 2 CS peptides (CoV2 residues 617-672, HKU1 residues 757-812) that did not share amino acid homology was uncorrelated, except for in highly neutralizing CCP that often reacted to both. The 2 RBD peptides (CoV2 residues 533-588, HKU1 residues 617-672) had a moderate level of amino acid homology and were frequently coreactive, but only the High NT CCP had a nonzero correlation between fold changes for CoV2 RBD and HKU1 RBD (Pearson's correlation, $P<0.001$ ). The CoV2 and HKU1 RBD peptide reactivities were sometimes independently reactive; recognition of RBD peptides from both viruses may reflect cross-reactivity and/or independent, non-crossreactive (yet correlated) reactivities. These examples highlight the complex interplay between preexisting, boosted, and de novo cross-reactive antibody responses to coronaviruses.

Deconvoluting antibody specificity from VirScan data. We hypothesized that an individual's $\mathrm{HCoV}$ immune response history might impact the spectrum of epitopes targeted during CoV2 infection and the resultant CCP functionality. Clearly, however, many $\mathrm{HCoV}$ reactivities observed in the CCP are likely due to crossreactive CoV2 antibodies, as we described previously (12). We therefore sought to deconvolute the VirScan data, assuming that antibodies would exhibit preference for their intended on-target peptides compared with cross-reactive peptides (see Methods, Deconvolution; and schematic in Supplemental Figure 5). Deconvoluted profiles retain only definitive target-preferred reactivities.

The deconvoluted VirScan prevalence data are plotted on the coronavirus genomes in Figure 4A. As expected, Pre-COVID CoV2 reactivity largely disappeared, since these samples can only harbor cross-reactive antibody binding to CoV2. Additionally, many $\mathrm{CoV} 2 \mathrm{~S}$ reactivities highly correlated with homologous $\mathrm{HCoV}$ peptide reactivities, such as the FP reactivities, were reduced due to insufficient support for preference of a specific coronavirus. Some reactivities against the $\mathrm{CoV} 2 \mathrm{~S} \mathrm{RBD}$ and $\mathrm{HR} 2$ were also removed, whereas reactivities around the CoV2 S CS region were retained (Figure 4B, dominant region 3). N peptides, meanwhile, tended to be more readily distinguishable as non-cross-reactive. Most reactivities against $\mathrm{OC} 43$ (the $\mathrm{HCoV}$ most closely related to CoV2; ref. 13) were either unattributable or attributed to antibodies targeting CoV2, and were thus removed by deconvolution. Aggregated, deconvoluted $\mathrm{CoV} 2$ and $\mathrm{HCoV}$ reactivities were then assessed for correlation with plasma functionality (Figure 4, C and D). As expected, NT AUC remained highly correlated with aggregated, deconvoluted CoV2 reactivity, while the OC43 association with NT AUC was no longer supported by deconvoluted data. After deconvolution, the increased NL63 score remained associated with higher NT AUC (Wilcoxon's test, $P=0.005$ ), further supporting a role for prior NL63 infection in elevated CoV2 NT AUC.

We next evaluated associations between individual deconvoluted peptide-level reactivities with plasma functionality (Supplemental Table 2). The most immunodominant CoV2 S RBD peptide (residues 533-588) was preferentially targeted in $18.8 \%$ of High NT CCP compared with $10.3 \%$ of Medium NT CCP, and only $1.8 \%$ of Low NT CCP (Fisher's, High NT vs. Low NT $P$ $=0.009$ ). In contrast, HKU1-preferred RBD (residues 617-672) antibodies were identified in $40.6 \%$ of High NT CCP and $50.9 \%$ of Low NT CCP (difference not significant). These results indi- 
Table 1. Immunodominant coronavirus peptides and their functional correlates

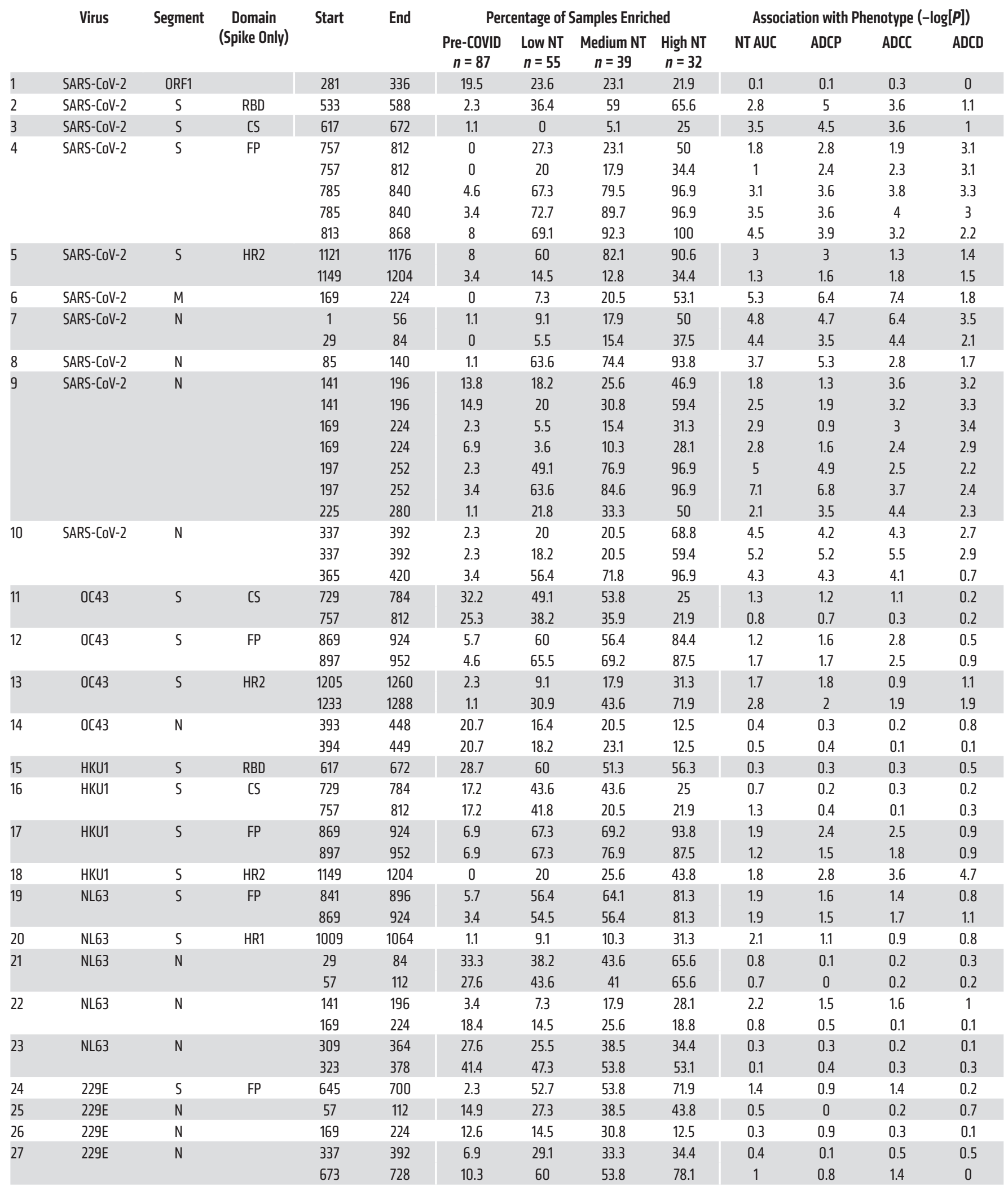

Fifty-two peptides defining 27 immunodominant regions were identified. The percentage of samples with a specific reactivity is shown. Associations with COVID-19 convalescent plasma functionality were defined by dichotomizing all convalescent plasma as positive or negative for a particular reactivity, followed by a 2-sided Wilcoxon test. The negative-log-transformed $P$ values are shown. Adjusted significance cutoff was 0.0255 or, negative-logtransformed, 1.59 (Benjamini-Hochberg). 
A
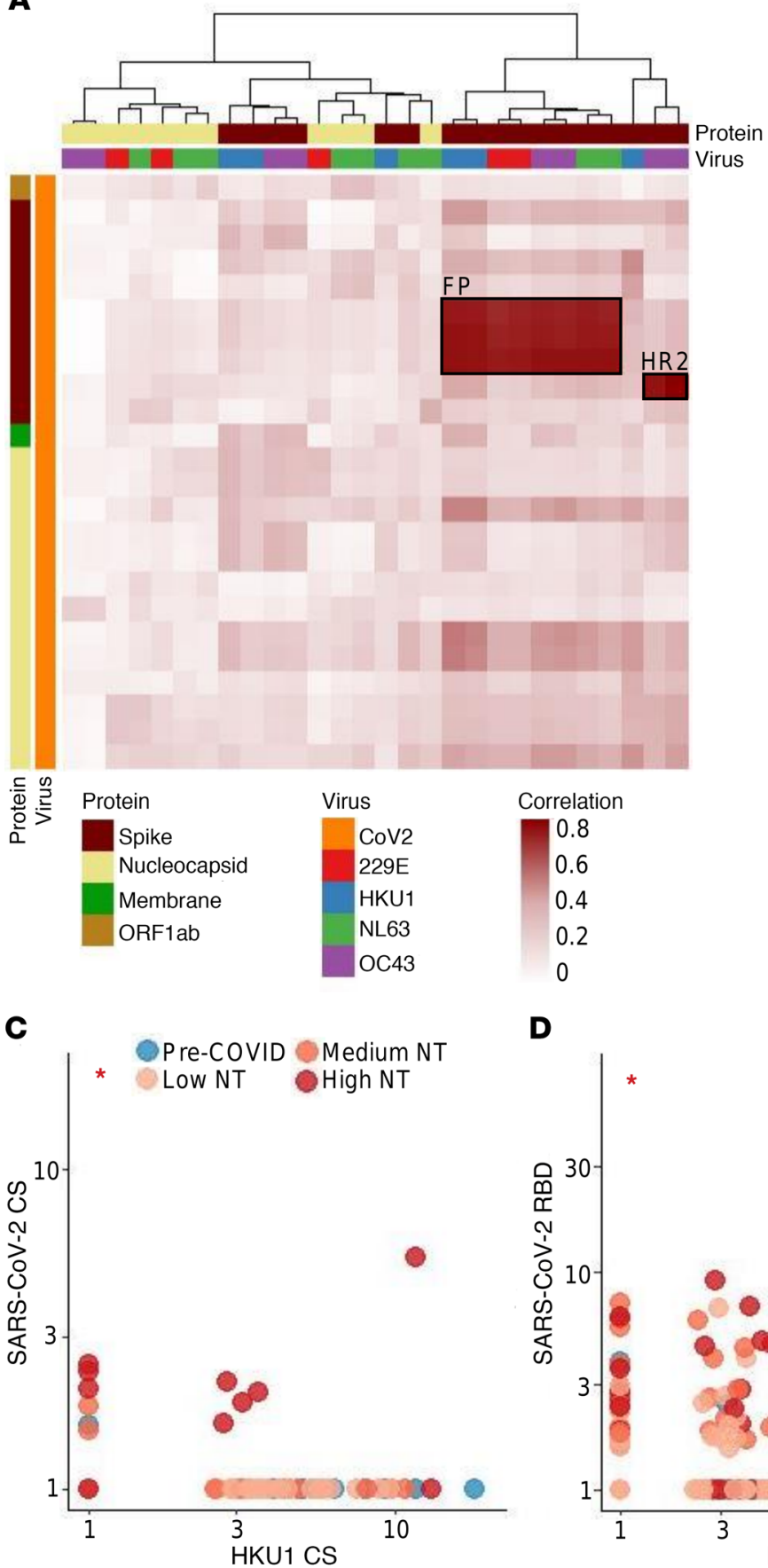

B

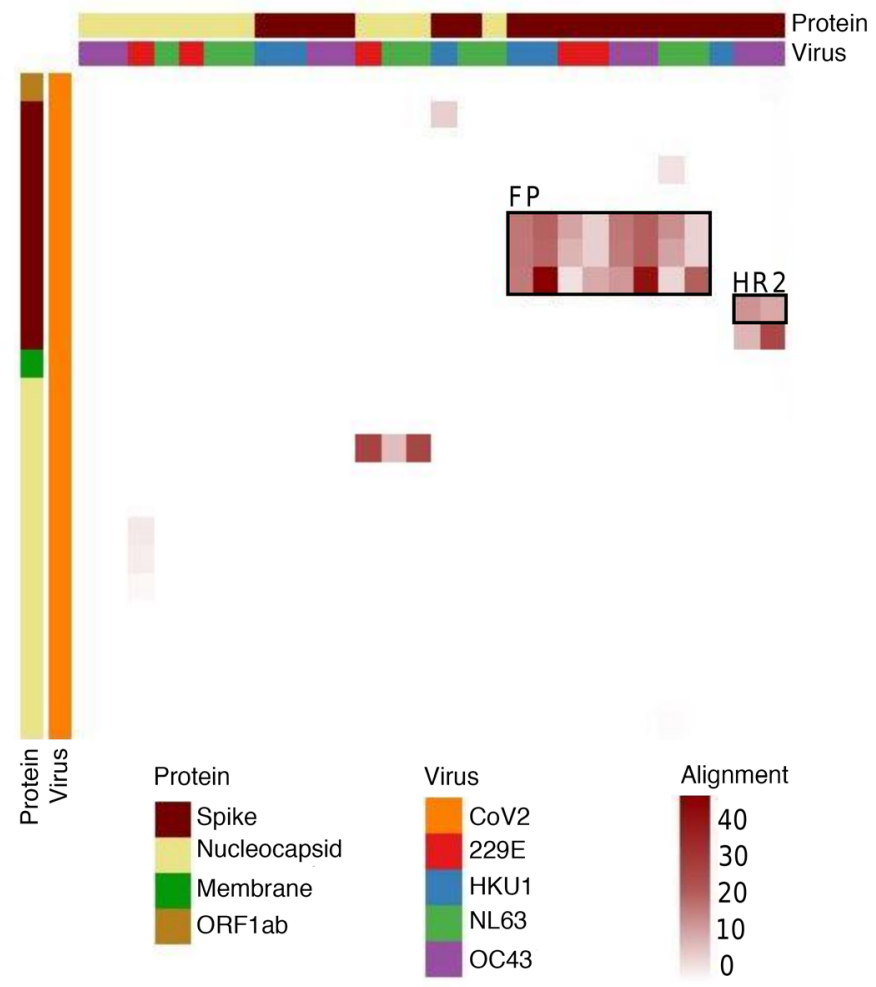

D

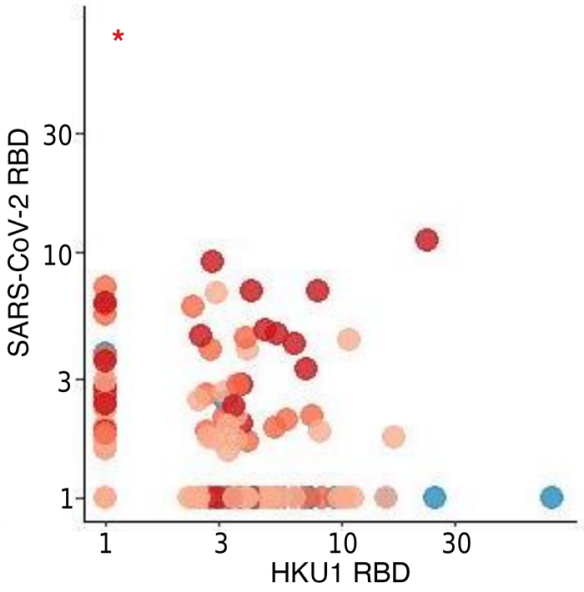

$\mathbf{E}$

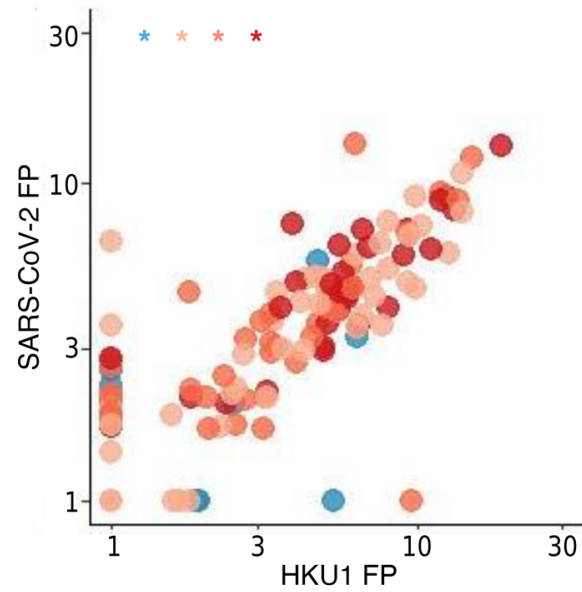

Figure 3. Reactivities against some CoV2 peptides are highly correlated with reactivities against homologous HCoV peptides. (A) Spearman's correlation coefficient matrix between dominant CoV2 peptides and dominant HCoV peptides is shown in the form of a clustered heatmap. CoV2 peptides ( $y$ axis) are ordered by genomic location from top to bottom, while HCoV peptides ( $x$ axis) are clustered according to their correlations. The heatmap annotations depict peptides' protein of origin and virus of origin. Highly correlated peptides map to fusion peptide (FP) or heptad repeat 2 (HR2). (B) Sequence similarity (defined by the negative log of the blastp $E$ value) between dominant CoV2 peptides and dominant HCoV peptides is shown. The rows and columns of the heatmap match those of the correlation heatmap to facilitate comparison. The regions of highest correlation (boxes as in $\mathbf{A}$ ) show strongest alignment. (C-E) Antibody reactivity (measured as fold changes) to 3 HKU1 S peptides are plotted against reactivity to homologous peptides of CoV2 S. The 2 CS peptides have no sequence homology and their reactivity is not correlated. The 2 RBD peptides have moderate homology and show frequent coreactivity but no strong correlation. The 2 FP peptides have high sequence homology and strong correlation among all sample groups. Asterisks indicate Pearson's correlation with nonzero coefficient for a given plasma group $(P<0.05)$. 
A
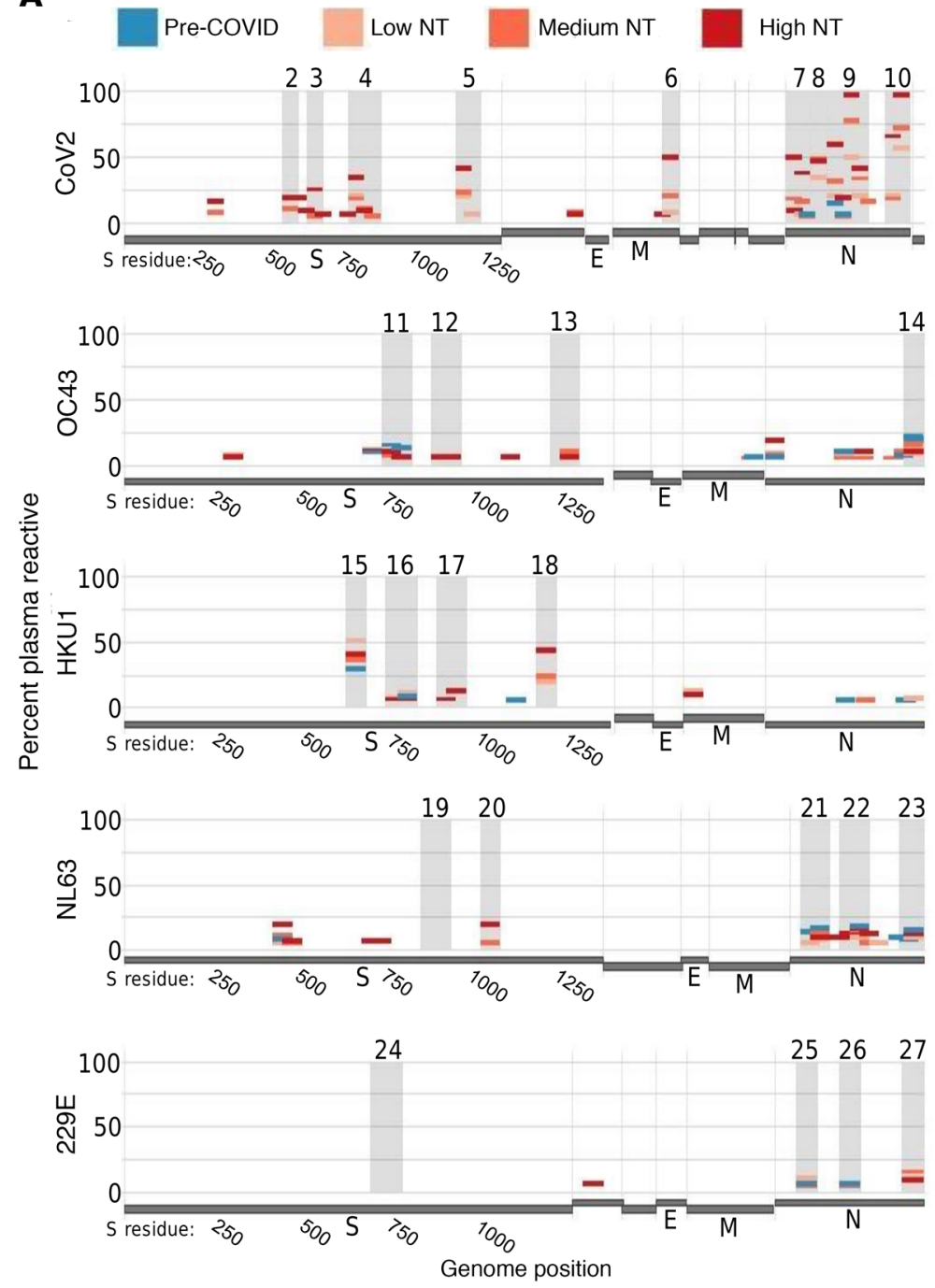
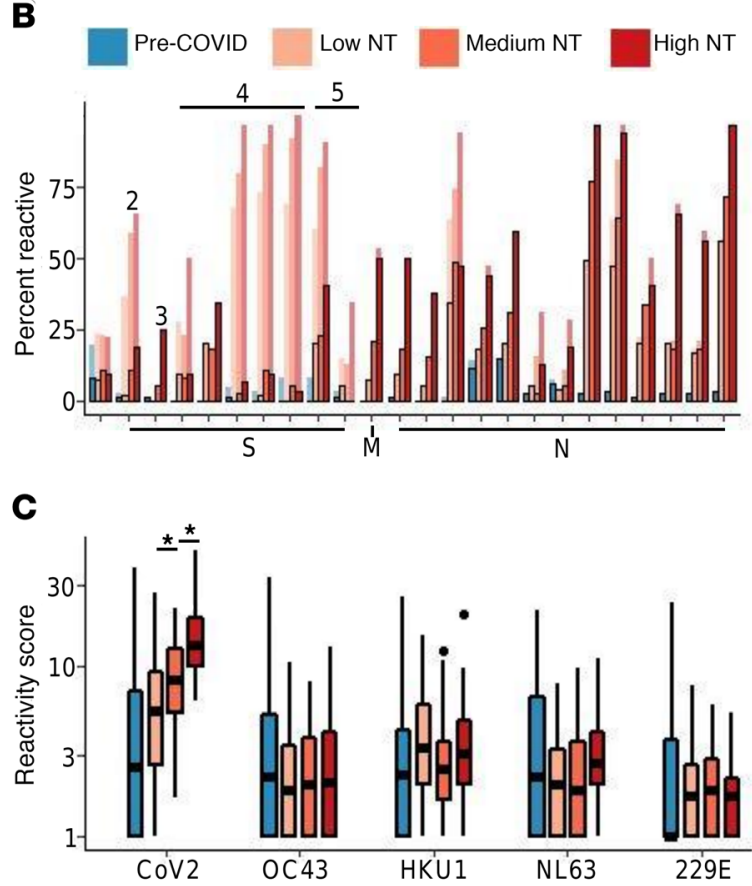

D

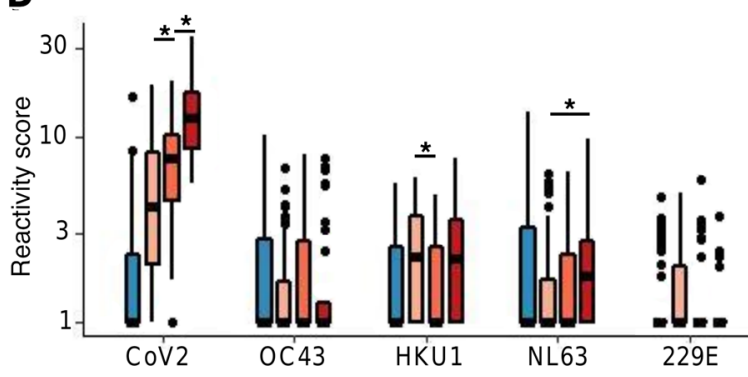

Figure 4. Deconvolution of COVID-19 convalescent plasma reactivities. (A) Following deconvolution, the percentage of samples in each sample group with target-preferred peptide reactivities were plotted along the viral genomes. Amino acid residue number is included for the spike protein (S). Pre-COVID, $n=$ 87; Low NT, $n=55$; Medium NT, $n=39$; and High NT, $n=32$. Cene products: spike (S), envelope (E), membrane (M), and nucleocapsid (N). (B) The percentage of plasma in each sample group that had reactivity to dominant CoV2 peptides is shown before (light bars) and after (dark bars with outline) deconvolution. (C and $\mathbf{D})$ Aggregate virus scores were calculated following deconvolution, using all peptides (C) or using only peptides from immunodominant regions (D). ${ }^{*} P<0.05$ by 2 -sided Wilcoxon's test, indicating COVID-19 convalescent plasma groups that show significantly different scores.

cate that CoV2-preferred antibody reactivity to RBD, in contrast with HKU1-preferred antibody reactivity to RBD, is associated with higher NT AUC.

Discordance of CoV2 S antibody titer versus NT AUC. The suitability of CCP donations is typically evaluated by measuring total $\mathrm{S}$ antibody titer using an enzyme-linked immunosorbent assay (ELISA). Total $\mathrm{S}$ antibodies correlate with neutralization in our cohort and others $(8,9,20)$. However, $S$ antibodies tend to be more sensitive than specific for neutralization. Using VirScan, we investigated whether reactivities to specific peptides were associated with discordance between functionality and total $S$ reactivity.

For this analysis, we performed a simple linear regression to predict NT AUC using total S titer determined by a multiplex microsphere assay (13). CCP samples were then categorized into 3 groups: High NT/Low S, Concordant NT/S, and Low NT/High
$\mathrm{S}$ (Figure 5A and Methods). To evaluate whether boosting of nonneutralizing $\mathrm{HCoV}$ antibodies contributed to lower NT/S ratios, we first tested for associations between aggregate coronavirus scores and NT/S discordance (Figure 5, B and C). We found a correlation between aggregate HKU1 score and Low NT/High $\mathrm{S}$ (predeconvolution Wilcoxon's, $P=0.019$; postdeconvolution Wilcoxon's, $P=0.011$ ). We then explored whether reactivity to specific HKU1 peptides could account for this association. A single HKU1 peptide, the previously identified CS peptide (residues 757-812, dominant region 16 in Table 1), was significantly associated with Low NT/High S (Wilcoxon's, $P=0.023$ ). The association between HKU1 S CS reactivity and overprediction of NT AUC depended on the absence of CoV2 CS (residues 617-672) reactivity (Figure 5D). When reactivities to HKU1 CS and CoV2 CS peptides were taken into account, roughly half of the samples with Low NT/S were identified as less suitable for transfusion 


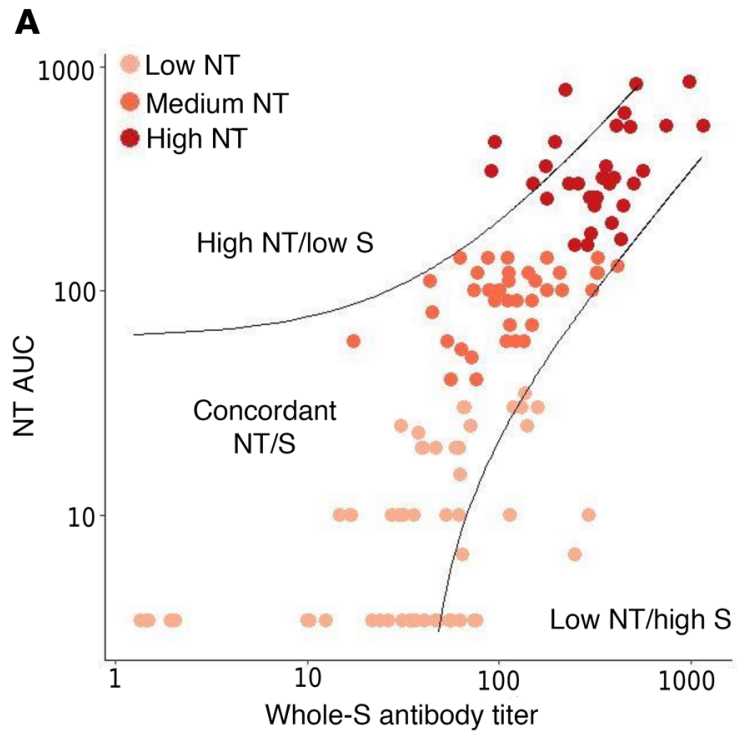

B
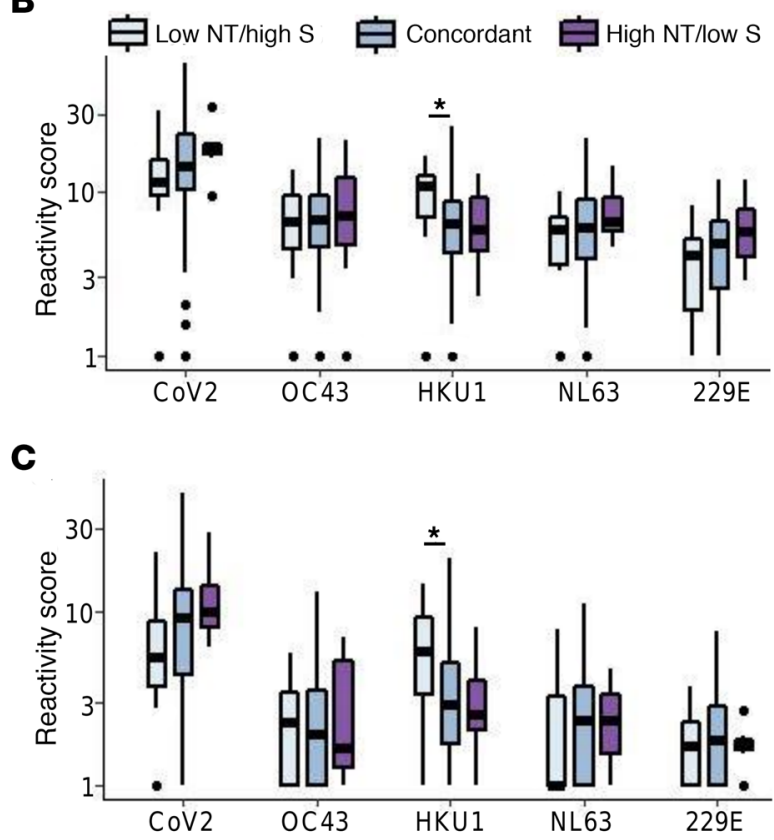

D

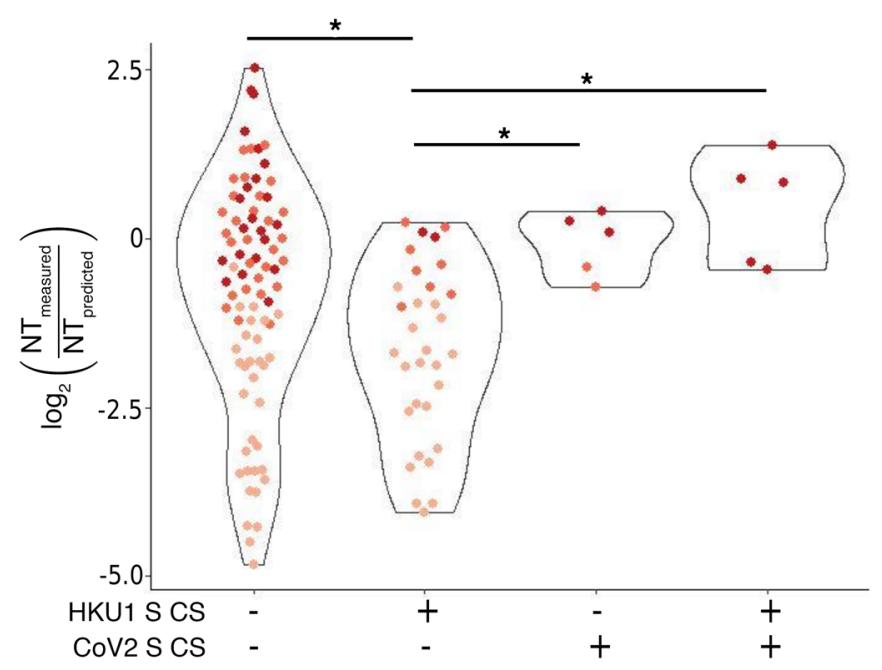

$\mathbf{E}$

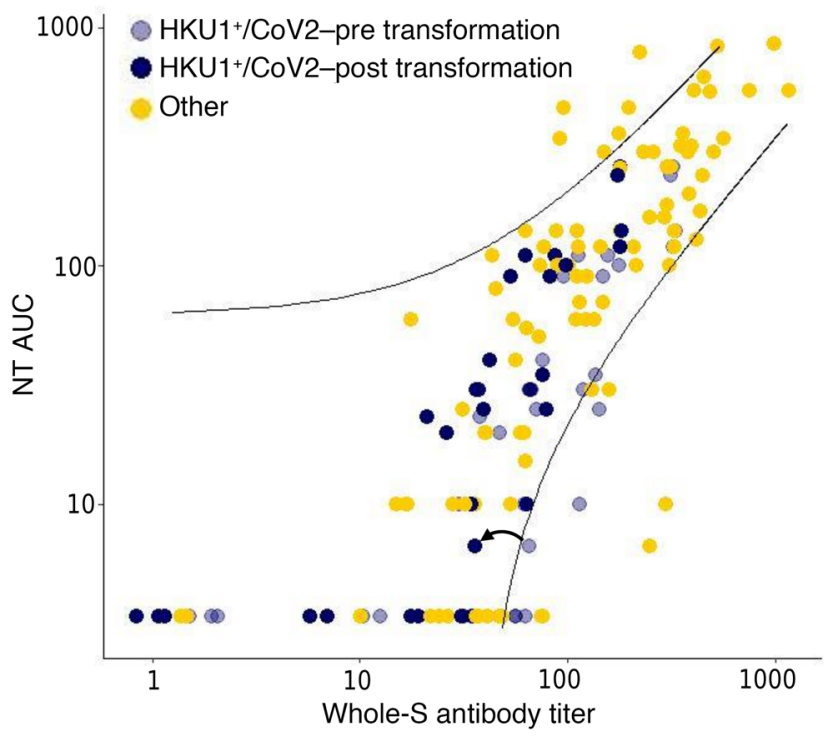

Figure 5. VirScan identifies features associated with discordance between whole-spike titer and neutralizing titer. (A) NT AUC was plotted against whole-S antibody titer. A linear regression was performed between whole S and NT AUC to establish a predicted NT AUC; boundary lines were plotted to indicate samples displaying large discordance between NT AUC and whole-S titer (Methods). Low NT, $n=55$; Medium NT: $n=39$, and High NT: $n=32$. (B and C) Aggregate virus scores were calculated for COVID-19 convalescent plasma with concordant NT/S $(n=106)$, discordantly Low NT/S ( $n=13$ ), and discordantly High NT/S $(n=7)$ using predeconvolution (B) and postdeconvolution (C) peptide reactivity. Horizontal bars indicate differences in scores between groups. ${ }^{*} P<0.05$ by Wilcoxon's rank sum test. (D) Ratio of measured NT AUC to predicted NT AUC, versus CoV2 CS and HKU1 CS reactivities. ${ }^{*} P<0.05$ by 2-sided Wilcoxon's test. (E) COVID-19 convalescent plasma defined by the HKU1 CS+/CoV2 CS- reactivity pattern $(n=32)$ are shown on the scatter plot in $\mathbf{A}$ A correction factor (example indicated by arrow) was applied to these plasma samples to account for the association with NT/S discordance.

(Figure 5E and Methods). These results establish that the fine specificities of anti-coronavirus antibodies, including $\mathrm{HCoV}$ reactivities, may prove helpful in improving the assessment of CCP functionality for clinical use.

\section{Discussion}

In this study, we used VirScan to quantify the relationship between the fine specificities of anti-coronavirus antibodies and the functionality of CCP. These analyses allowed us to define peptide epitopes targeted by preexisting antibodies that cross-react with CoV2. Many antibody reactivities to $\mathrm{HCoV}$ peptides observed in the convalescent phase of CoV2 infection likely reflect an anamnestic response (boosting of preexisting antibody reactivities by $\mathrm{CoV} 2)$ or a feature of the cross-reactive $\mathrm{CoV} 2$ antibody response, as previously described (12). In either case, $\mathrm{HCoV}$ reactivities represent potential biomarkers for identifying $\mathrm{CCP}$ with differing therapeutic potency. In particular, polyclonal responses to CoV2 and NL63, as well as a cross-reactive response to the conserved 
FP of CoV2 S, were all associated with increased plasma functionality. In contrast, CCP with antibodies that recognize HKU1 CS peptides but not CoV2 CS peptides suffered from relatively low neutralizing activity.

The defining feature of highly neutralizing CCP was high polyclonality of CoV2 antibodies. High NT CCP reacted with most of the same immunodominant epitopes as the low NT CCP, and at relatively similar levels of reactivity, but at much higher frequency. NT, ADCC, ADCP, and ADCD have been found to correlate with one another (13), but individual peptide epitopes displayed variable associations with different, sometimes unexpected, functionalities. It is important to recognize that antibody reactivity to individual peptides may track with increased CCP functionality for 2 opposing reasons. First, they may be positively correlated with disease severity (since disease severity is positively correlated with convalescent CCP functionality). Alternatively, reactivities may directly contribute to a more effective humoral response (thus tending to negatively correlate with disease severity). Antibodies that react to the immunodominant RBD peptide, FP peptides, and the CS peptide are the most likely antibodies described in this study to be neutralizing. Support for the functional relevance of CS peptide-specific antibodies derives from escape mutations in this region that have arisen in response to therapeutic antibodies (21). However, the relationship between reactivity to the CS region and disease severity is not known. Beyond CoV2 antibody specificities, aggregation of postdeconvolution NL63 peptide reactivities suggested that a stronger immune response to NL63 is associated with generating highly neutralizing CCP. This result is consistent with the previously reported association between recent $\mathrm{HCoV}$ infection and milder COVID-19 disease severity (22) as well as the correlation between NL63 neutralization and milder disease severity (16), the latter case being potentially attributable to NL63's usage of angiotensin-converting enzyme 2 (ACE2) for cell entry, as is the case for CoV2.

Preexisting antibodies that react with CoV2 S2 have been described; however, data on the activity of these antibodies are conflicting $(14,23)$. We had previously shown that reactivity to the highly conserved FP region might explain preexisting antibody reactivity to CoV2 S2 and that FP represents a pan-coronavirus conserved antibody epitope (12). The FP and HR2 regions of CoV2 $\mathrm{S}$ are likely the most dominant for 2 main reasons. First, they have high sequence homology to other HCoVs and can therefore elicit memory responses. Second, being less conformational and less posttranslationally modified, the associated epitopes are more readily displayed on phage. In this study, a small minority of Pre-COVID plasma was found to contain antibodies reactive to CoV2 FP peptides ( $\leq 10 \%$, similar to CoV2 HR2 reactivity). Deconvolution attributed almost all of the observed PreCOVID reactivity against CoV2 FP and HR2 to cross-reactivity from antibodies that recognize $\mathrm{HCoV}$ peptides. The cross-reactive response to $\mathrm{HCoV}$ FP and CoV2 FP appears to be boosted by CoV2 infection, and while support for or against the functionality of these antibodies is currently lacking, their correlation with increased CCP functionality is consistent with a protective role. Our findings suggest that the pooled prepandemic plasma used to make intravenous immunoglobulin (IVIG) likely contains low levels of antibodies reactive to CoV2 FP and/or HR2.
If these antibodies are found to neutralize CoV2, prepandemic IVIG would thus likely contain a low level of neutralizing activity. If anti-FP antibodies directly confer protection, banked plasma containing anti-FP antibodies may also prove useful in future coronavirus outbreaks (including zoonotic coronaviruses related to the bat coronaviruses in our library) and FP would represent a candidate antigen for universal coronavirus vaccination.

Therapeutic use of CCP transfused early in infection and with a higher titer of anti-CoV2 antibodies is more likely to be efficacious (6). However, efficacy data for CCP are mixed. Although the FDA initially recommended measuring neutralizing titers for CCP, the low capacity of BSL-3 laboratories nationwide limits the ability to quantify viral neutralization at the scale required for widespread use. Surrogate assays that measure neutralizing antibodies without requiring a BSL-3 lab are in development (24), but ELISA-based measurements of antibodies directed against S, S1, or RBD are currently used. We evaluated the specific peptide reactivities associated with the greatest relative difference between whole-S antibody titer and neutralizing titer. HKU1 reactivity, specifically directed against the CS peptide, was associated with relatively low functionality. We note that a limited number of CoV2 CS-reactive CCP were identified in this study. The utility of the 2 peptide serosignatures will therefore need to be validated in an independent cohort. If confirmed, evaluating CCP for reactivity to HKU1 S CS and CoV2 S CS would represent a potential strategy to identify CCP less likely to have clinical benefit.

IgG antibodies in CCP are at significantly higher levels than IgA or IgM and are thought to confer the majority of the therapeutic effect (8). Additionally, IgG antibodies are likely the primary mediators of in vitro viral neutralization (25). We have therefore used combined protein A- and protein $\mathrm{G}$-coated magnetic beads to capture all IgG subclasses (IgG1-IgG4) for VirScan analysis. Although mucosal IgA is known to play an important role in protection from reinfection, it is not captured well by protein A or $\mathrm{G}$, and is thus unlikely to have contributed to the VirScan data described here. IgM, meanwhile, may contribute to measured CoV2 neutralization and/or ADCD, but is also poorly bound by protein A or G. Epitopes targeted preferentially by IgA or IgM that associate with plasma antibody functionality may therefore be underrepresented in our study.

We identified specific $\mathrm{HCoV}$ antibody responses that are likely to impact the potency of CCP. Our analyses are most immediately relevant to lineages of CoV2 that are not antigenically different than the SARS-CoV-2/USA-WA1/2020 strain. Novel CoV2 strains have recently emerged and their antigenicity has not been fully characterized. Future epitope versus NT studies will need to carefully consider potential antigenic mismatch as a confounding variable. We additionally developed a method using VirScan data to distinguish preferential antibody recognition from ambiguous cross-reactivity, which may be used more broadly for other viruses to better understand original antigenic sin, versus heterologous protection. This approach may also be used to augment current VirScan analysis strategies that seek to differentiate antibody responses among related viruses (26). An important limitation of VirScan is that the phage do not display highly conformational, discontinuous, or glycosylated epitopes. Some, if not most, of the antibody reactivities identified in this study are unlikely to confer func- 
tional activity to the plasma. However, these reactivities may track closely with functional antibody reactivities that are not detectable by VirScan. Regardless of whether HCoV peptide reactivities are preexisting or arise in the context of CoV2 infection, we found clear differences in $\mathrm{HCoV}$ reactivity between poorly and highly functional CCP, defined by capacity to neutralize CoV2, stimulate phagocytosis, stimulate cellular cytotoxicity, and fix complement. For example, highly neutralizing CCP contained a stronger polyclonal antibody response to NL63. Additionally, HKU1 S CS reactivity confounds prediction of NT AUC based on whole-S titer, while antibody preference for CoV2 RBD over HKU1 RBD identifies highly neutralizing CCP. An understanding of the fine specificities of anti-coronavirus antibody repertoires may serve to generate novel hypotheses regarding the molecular mechanisms underlying the complex immune responses elicited by CoV2 infection.

\section{Methods}

Study participants. Eligible CCP donors were contacted by study personnel, as previously described (8). All donors were at least 18 years old and had a confirmed diagnosis of CoV2 by detection of RNA in a nasopharyngeal swab sample. In addition, donors were informed that they would need to satisfy standard eligibility criteria for blood donation (e.g., not pregnant within the last 6 weeks, never been diagnosed or have risk factors for transfusion-transmitted infections such as HIV, hepatitis $\mathrm{B}$ virus, or hepatitis $\mathrm{C}$ virus). Basic demographic information (age, sex, hospitalization with COVID-19) was obtained from each donor; initial diagnosis of CoV2 and the date of diagnosis were confirmed by medical chart review. All donors provided informed consent and approximately $25 \mathrm{~mL}$ of blood was collected in acid citrate dextrose (ACD) tubes. The samples were separated into plasma and peripheral blood mononuclear cells within 12 hours of collection. The plasma samples were immediately frozen at $-80^{\circ} \mathrm{C}$.

To test samples prior to the COVID-19 pandemic, stored plasma specimens from an identity-unlinked HIV serosurvey conducted in 2016 among adult patients attending the Johns Hopkins Hospital Emergency Department were included $(n=87)$. Prepandemic specimens were excess (i.e., discarded) samples from patients who had blood drawn for clinical purposes (27).

Both parent studies were cross-sectional and no individual contributed multiple specimens.

Virus and neutralization assay. The SARS-CoV-2/USA-WA1/2020 virus was obtained from BEI Resources, NIAID, NIH. The virus was grown and infectious virus titrated on VeroE6TMPRSS2 cells, provided by the National Institute of Infectious Diseases, Tokyo, Japan. Viral titration occurred at $33^{\circ} \mathrm{C}$ in infection media, which was identical to the media used to grow the cells except the FBS was reduced to $2.5 \%$, as described previously for CoV2 (28).

Plasma neutralization assays were performed essentially as previously described $(8,29)$. CoV2 was added to serially diluted plasma, and the plasma-virus mixture was incubated with VeroE6TMPRSS2 cells (30). Cytopathic effect was scored following $4 \%$ formaldehyde fixation and staining with Napthol Blue-Black, and an AUC, representing plasma neutralizing activity, was determined.

Phagocytosis assay. ADCP was assessed as previously described (13). Briefly, uptake of fluorescent RBD-conjugated microspheres by monocytic human THP- 1 cells in the presence of CCP was defined by flow cytometry.
CD16 reporter assay. FcgRIIIa ligation activity was defined as previously reported (13) as a surrogate of ADCC activity. The ability of CCP to induce luciferase expression in a Jurkat reporter cell line via ligation of FcgRIIIa was defined by culturing on RBD-coated high-binding microtiter plates in the presence of CCP.

Complement deposition assay. ADCD of heat-inactivated CCP was quantified as previously described (13) by incubation of RBDconjugated multiplex assay microspheres in the presence of human complement serum and subsequent quantification of complement cascade product $\mathrm{C} 3 \mathrm{~b}$ deposition by flow cytometry using murine antiC3b (Cedarlane, CL7636AP) and anti-mouse IgG1-PE secondary antibody (Southern Biotech, 1070-09).

Luminex assessment of whole-S IgG. Prefusion-stabilized, trimerforming spike protomers (S-2P) of CoV2 were expressed in Expi 293 cells, purified via affinity chromatography, and covalently coupled to Luminex Magplex magnetic microspheres, as previously described (13). Antigen-specific antibodies were detected with secondary antiIgG-PE (Southern Biotech, 9040-09) and median fluorescence intensity was measured on a FlexMap 3D array reader.

Programmable phage display immunoprecipitation and sequencing. The design and cloning of the 56-amino acid coronavirus libraries were previously described (12). Phage immunoprecipitation and sequencing were performed according to a previously published protocol (31). Briefly, $0.2 \mu \mathrm{L}$ of each plasma sample was individually mixed with the coronavirus phage library and immunoprecipitated using protein A- and protein $\mathrm{G}$-coated magnetic beads. A set of 6-8 mock immunoprecipitations (no plasma input) were run on each 96-well plate. Bead washing was implemented on a Bravo liquid handling robot. Magnetic beads were resuspended in PCR master mix and subjected to thermocycling. A second PCR reaction was employed for sample barcoding. Amplicons were pooled and sequenced on an Illumina NextSeq 500 instrument.

Statistics. Sequencing reads were mapped to library peptides using exact matching. A Bayesian hierarchical model was used to analyze the read count data and infer peptide enrichment. The read counts for each peptide were modeled as $\operatorname{binomial}(n, P)$ distributions based on the total sample read counts (depth, $n$ ) and probabilities $(P)$ drawn from $\beta$ distributions. Posterior distributions for enrichment were based on 10,000 iterations derived from JAGS (Just Another Gibbs Sampler) with slice sampling as implemented in R (using a binary yes/no indicator for enrichment in the chain), comparing the sample read counts to those observed in the mock immunoprecipitations. Reported fold changes are conditional on enrichment status; fold changes for peptides with enrichment posterior probability less than $50 \%$ are reported as 1 , and otherwise as the average fold change among the states where the above-mentioned binary indicator in the chain indicated enrichment (by definition, more than 5,000 iterations). Each peptide in the coronavirus VirScan library is represented by duplicate peptides, distinguishable by unique codon usage. The greater of the 2 fold changes of the technical replicate peptide pairs was used for downstream analyses.

Differences among virus-specific scores between sample groups were assessed via 2-sided Wilcoxon's rank sum tests. Wilcoxon's rank sum tests were additionally used to evaluate differences in functionality of CCP with, versus without, particular peptide enrichments. Correlations between number of reactivities versus NT AUC, as well as between CoV2 and HKU1 peptides, were determined via Pearson's 
correlation. Differences in enrichment rate between sample groups was calculated with Fisher's exact test. When multiple tests were used, significance was determined via the Benjamini-Hochberg procedure. All analyses and visualizations were performed in R (32), supported by R package pheatmap (33).

Deconvolution of VirScan data. The deconvolution algorithm to infer on-target reactivity versus off-target cross-reactivity was written in R. It was designed to determine the true targets of antibodies detected with VirScan under the assumption that reactivity (fold change of peptide enrichment) between an antibody and its true target will exceed that of off-target cross-reactivity. First, all of the 56-amino acid peptides from CoV2 or the endemic coronaviruses in the VirScan library were aligned with blastp. Peptides from the same protein of origin that had alignments with an $E$ value of less than 100 were considered potentially cross-reactive. Supplemental Figure 6 shows the peptide-peptide sequence homology map from blastp alignment of all CoV2 S, M, and N peptides against all $\mathrm{HCoV}$ S, M, and N peptides. Peptides were visualized as nodes and were colored according to the virus from which they were derived; peptides were linked if they shared alignments with an $E$ value of less than 100. For a peptide of interest (peptide A), the reactivity was compared to the reactivities of peptides from different coronaviruses that aligned to peptide A (comparison peptides). Binding "preference" required the fold change of peptide A be greater than the fold changes of all comparison peptides plus a factor accounting for technical dispersion unique to each peptide pair. In order to establish the dispersion factor, we took advantage of the fact that each peptide in the library is represented in duplicate. The standard deviation of the difference between technical replicate peptides was used as a measure of expected variance in peptide reactivity, and the dispersion factor was set at 2 times this standard deviation for each peptide pair. Supplemental Figure 5 contains a flow chart describing the deconvolution algorithm.

Neutralizing titer and whole-S antibody titer discordance analysis. If NT AUC predicted by a simple linear regression was greater than 40 plus 2 times the measured NT AUC, the sample was said to have discordantly High S. Similarly, if a sample's measured NT AUC was greater than 40 plus 2 times predicted NT AUC, it was said to have discordantly Low $\mathrm{S}$.

The HKU1 S CS$+\mathrm{CoV} 2 \mathrm{~S} \mathrm{CS}^{-}$transformation was designed to normalize the error in predicted NT AUC. The $\log _{2}$ of the median measured NT AUC to predicted NT AUC ratio for $\mathrm{HKU}_{\mathrm{S} \mathrm{CS}^{+}} /$ CoV2 S CS${ }^{-}$samples was -0.72 , while the $\log _{2}$ of the median measured NT AUC to predicted NT AUC ratio for all other samples was -1.67. We normalized this ratio for the samples that were HKU1 CS positive and Cov2 CS negative to the ratio for all other samples to account for this difference.

Study approval. Written informed consent was received from all study participants. The Johns Hopkins University School of Medicine Institutional Review Board reviewed and approved the sample collection and this study.

\section{Author contributions}

WRM designed and performed data analysis and wrote the manuscript. SNH designed and conducted experiments and edited the manuscript. DRM designed and performed data analysis and edited the manuscript. A Chen processed raw data and edited the manuscript. EMB collected and managed samples and edited the manuscript. EF designed and provided reagents. IR designed analyses and edited the manuscript. KL, ARC, HN, SE Butler, and $\mathrm{AP}$ designed and performed experiments and edited the manuscript. JAW managed data and samples and edited the manuscript. MZL performed experiments and provided reagents. TSB, SE Benner, AB, SS, DS, and OL procured samples and edited the manuscript. TCQ procured samples and edited the manuscript. SHE and A Casadevall advised research and edited the manuscript. ADR conceived the project and edited the manuscript. MEA supervised research and edited the manuscript. SJE oversaw reagent development, provided reagents, and edited the manuscript. MR supervised the research and wrote the manuscript. AART collected samples, conceived the project, and edited the manuscript. HBL conceived the project, supervised research, and wrote the manuscript.

\section{Acknowledgments}

This study was made possible by grants from the Johns Hopkins COVID-19 Research Response Program, National Institute of General Medical Sciences grant R01GM136724 and NIAID grant U24AI118633 (to HBL); NIAID grants R01AI120938, R01AI120938S1, and R01AI128779 (to AART); NIH grants AI052733, AI15207, and HL059842 (to AC); in part by the Division of Intramural Research, NIAID, NIH (to OL, AR, and TCQ); National Heart Lung and Blood Institute grant 1K23HL151826-01 (to EMB); Bloomberg Philanthropies (to AC); Department of Defense grant W911QY2090012 (to DS); NIH Center of Excellence for Influenza Research and Surveillance grant HHSN272201400007C (to AP); the MassCPR and VOVRN (to SJE); and HIV Prevention Trials Network grant UM1AI068613 (to SHE). We are grateful to the National Institute of Infectious Diseases, Japan, for providing VeroE6TMPRSS2 cells and acknowledge the Centers for Disease Control and Prevention, BEI Resources, NIAID, NIH for providing SARS-related coronavirus 2, isolate USA-WA1/2020, NR-5228.

Address correspondence to: H. Benjamin Larman, Miller Research Building, Room 607, Baltimore, Maryland 21205, USA. Phone: 410.614.6525; Email: hlarman1@jhmi.edu. Or to: Matthew Robinson, 600 N. Wolfe St., Phipps Building, Room 528, Baltimore, Maryland 21287, USA. Phone: 410.287.2443; Email: mrobin85@ jhmi.edu. Or to: Aaron A. R. Tobian, Carnegie Building, Room 437, Baltimore, Maryland 21287, USA. Phone: 443.287.0527; Email: atobian1@jhmi.edu.
1. Dong E, et al. An interactive web-based dashboard to track COVID-19 in real time. Lancet Infect Dis. 2020;20(5):533-534.

2. Bloch EM, et al. Promoting access to COVID-19 convalescent plasma in low- and middleincome countries [published online Septem- ber 20, 2020]. Transfus Apher Sci. https://doi. org/10.1016/j.transci.2020.102957.

3. Bloch EM, et al. Deployment of convalescent plasma for the prevention and treatment of COVID-19. J Clin Invest. 2020;130(6):2757-2765.

4. Duan K, et al. Effectiveness of convalescent plasma therapy in severe COVID-19 patients. Proc Natl Acad Sci U S A. 2020;117(17):9490-9496.

5. Marovich M, et al. Monoclonal antibodies for prevention and treatment of COVID-19. JAMA. 2020;324(2):131-132.

6. Joyner MJ, et al. Convalescent plasma antibody 
levels and the risk of death from Covid-19 [published online January 13, 2021]. N Engl JMed. https://doi.org/10.1056/NEJMoa2031893.

7. Libster R, et al. Early high-titer plasma therapy to prevent severe Covid-19 in older adults [published online January 6, 2021]. https://doi. org/10.1056/NEJMoa2033700.

8. Klein SL, et al. Sex, age, and hospitalization drive antibody responses in a COVID-19 convalescent plasma donor population. J Clin Invest. 2020;130(11):6141-6150.

9. Wang Y, et al. Kinetics of viral load and antibody response in relation to COVID-19 severity. J Clin Invest. 2020;130(10):5235-5244.

10. Benner SE, et al. SARS-CoV-2 antibody avidity responses in COVID-19 patients and convalescent plasma donors. J Infect Dis. 2020;222(12):1974-1984.

11. Zamecnik CR, et al. ReScan, a multiplex diagnostic pipeline, pans human sera for SARS-CoV-2 antigens. Cell Rep Med. 2020;1(7):100123.

12. Shrock E, et al. Viral epitope profiling of COVID-19 patients reveals cross-reactivity and correlates of severity. Science. 2020;370(6520):eabd4250.

13. Natarajan H, et al. SARS-CoV-2 antibody signatures robustly predict diverse antiviral functions relevant for convalescent plasma therapy [preprint]. https://doi.org/10.1101/2020.09.16.20196 154. Published on medRxiv September 18, 2020.

14. Ng KW, et al. Preexisting and de novo humoral immunity to SARS-CoV-2 in humans. Science. 2020;370(6522):1339-1343.

15. Henry C, et al. From original antigenic sin to the universal influenza virus vaccine. Trends Immu- nol. 2018;39(1):70-79.

16. Henss L, et al. Analysis of humoral immune responses in SARS-CoV-2 infected patients. JInfect Dis. 2021;223(1):56-61.

17. Patel EU, et al. Comparative performance of five commercially available serologic assays to detect antibodies to SARS-CoV-2 and identify individuals with high neutralizing titers. J Clin Microbiol. 2021;59(2):e02257-20.

18. Ferretti AP, et al. Unbiased screens show $\mathrm{CD} 8^{+} \mathrm{T}$ cells of COVID-19 patients recognize shared epitopes in SARS-CoV-2 that largely reside outside the spike protein. Immunity. 2020;53(5):1095-1107.e3.

19. Walls AC, et al. Structure, function, and antigenicity of the SARS-CoV-2 spike glycoprotein. Cell. 2020;181(2):281-292.

20. To KK, et al. Temporal profiles of viral load in posterior oropharyngeal saliva samples and serum antibody responses during infection by SARS-CoV-2: an observational cohort study. Lancet Infect Dis. 2020;20(5):565-574.

21. Baum A, et al. Antibody cocktail to SARSCoV-2 spike protein prevents rapid mutational escape seen with individual antibodies. Science. 2020;369(6506):1014-1018.

22. Sagar M, et al. Recent endemic coronavirus infection is associated with less-severe COVID-19. JClin Invest. 2021;131(1):143380.

23. Anderson EM, et al. Seasonal human coronavirus antibodies are boosted upon SARS-CoV-2 infection but not associated with protection [published online April 1, 2021]. Cell. https://doi. org/10.1016/j.cell.2021.02.010.

24. Tan CW, et al. A SARS-CoV-2 surrogate virus neutralization test based on antibody-mediated blockage of ACE2-spike protein-protein interaction. Nat Biotechnol. 2020;38(9):1073-1078.

25. Suthar MS, et al. Rapid generation of neutralizing antibody responses in COVID-19 patients. Cell Rep Med. 2020;1(3):100040.

26. Monaco DR, et al. Deconvoluting virome-wide antiviral antibody profiling data [preprint]. https://doi.org/10.1101/333625. Posted on bioRxiv May 30, 2018.

27. Kelen GD, et al. Improvements in the continuum of HIV care in an inner-city emergency department. AIDS. 2016;30(1):113-120.

28. Schaecher SR, et al. The ORF7b protein of severe acute respiratory syndrome coronavirus (SARS$\mathrm{CoV}$ ) is expressed in virus-infected cells and incorporated into SARS-CoV particles. J Virol. 2007;81(2):718-731.

29. Schaecher SR, et al. An immunosuppressed Syrian golden hamster model for SARS-CoV infection. Virology. 2008;380(2):312-321.

30. Matsuyama S, et al. Enhanced isolation of SARSCoV-2 by TMPRSS2-expressing cells. Proc Natl Acad Sci U S A. 2020;117(13):7001-7003.

31. Mohan D, et al. PhIP-Seq characterization of serum antibodies using oligonucleotide-encoded peptidomes. Nat Protoc. 2018;13(9):1958-1978.

32. $R$ : A language and environment for statistical computing. Version 4.0.2. R Foundation for Statistical Computing; 2020. Accessed February 9, 2021. https://www.R-project.org/.

33. pheatmap: Pretty Heatmaps. Version 1.0.12. Raivo Kolde; 2019. Accessed February 9, 2021. https:// CRAN.R-project.org/package=pheatmap. 